\title{
SELEÇÃO DA MELHOR CONFIGURAÇÃO DE POÇO DE PETRÓLEO PARA O DESENVOLVIMENTO DE UM CAMPO UTILIZANDO O MÉTODO AHP TOPSIS 2N
}

\author{
Danilo Colombo \\ Universidade Federal Fluminense \\ Rua Passo da Pátria, $n^{\circ}$ 156, Escola de Engenharia, São Domingos, Niterói/RJ \\ colombo.danilo@hotmail.com
}

Marcos dos Santos

Centro de Análises de Sistemas Navais / Instituto Militar de Engenharia

Praça Barão de Ladário s/n, Ilha das Cobras, Rua da Ponte, Ed. 23 do AMRJ, Centro, Rio de Janeiro/RJ

Praça General Tibúrcio, nº 80, Praia Vermelha, Urca, Rio de Janeiro/RJ

marcosdossantos doutorado uff@yahoo.com.br

Carlos Francisco Simões Gomes

Universidade Federal Fluminense

Rua Passo da Pátria, nº 156, Escola de Engenharia, São Domingos, Niterói/RJ

cfsg1@bol.com.br

\begin{abstract}
RESUMO
O objetivo deste artigo é apresentar os resultados obtidos na seleção da melhor configuração de um poço de petróleo offshore para o desenvolvimento de um campo de petróleo utilizando um método de apoio multicritério à decisão (AMD). A atividade de seleção de configuração de projeto de poços é fundamental para uma companhia petrolífera, impactando nos prazos, custos e riscos de um projeto de desenvolvimento. Por tratar-se de uma atividade complexa, geralmente envolve times multidisciplinares (geologia, reservatório, elevação e escoamento, sistemas submarinos, perfuração, completação etc.). O artigo apresenta três configurações pré-selecionadas por este time multidisciplinar e os critérios definidos para avaliar estas configurações. O método de AMD AHP-TOPSIS-2N foi aplicado para a definição da melhor configuração a ser utilizada.
\end{abstract}

Palavra-chave: Configuração de Poços; Multicritério; Tomada de Decisão; AHP-TOPSIS2N.

\begin{abstract}
The objective of this paper is to present the results obtained in the selection of the best configuration of an offshore well for the development of an oil field using a multi-criteria decision support (AMD) method. The activity of select well design configurations is critical for an oil company, affecting the timelines, costs and risks of a development project. Because it is a complex activity, it usually involves multidisciplinary teams (geology, reservoir, elevation and flow, subsea systems, drilling, completion etc.). The article presents
\end{abstract}


three configurations pre-selected by this multidisciplinary team and the criteria defined to evaluate these configurations. The AMD method AHP-TOPSIS-2N was applied to define the best configuration to use.

Keywords: Well Configurations; Multi criteria; Decision Making; AHP-TOPSIS-2N.

\section{Como Citar:}

COLOMBO, Danilo; SANTOS, Marcos dos; GOMES, Carlos Francisco Simões. Seleção da melhor configuração de poço de petróleo para o desenvolvimento de um campo: utilizando uma ferramenta de apoio multicritério. In: SIMPÓSIO DE PESQUISA OPERACIONAL E LOGÍSTICA DA MARINHA, 19., 2019, Rio de Janeiro, RJ. Anais [...]. Rio de Janeiro: Centro de Análises de Sistemas Navais, 2019.

\section{INTRODUÇÃO}

A recente queda no preço do barril do petróleo tem levado a indústria a buscar uma redução de custos nas atividades de exploração e produção, levando a projetos mais competitivos (PLEDGE et al., 2017). O desenvolvimento de um campo de petróleo offshore envolve diversos custos, como o custo da plataforma de produção, custos com dutos e linhas, custos com equipamentos submarinos e custos com poços. Os custos com a construção, manutenção e abandono de poços podem superar metade do custo total de desenvolvimento de um campo.

Os poços de petróleo submarinos são construídos utilizando sondas marítimas, cujo custo diário de aluguel pode chegar a mais de R\$ 1 milhão. Desta forma, o custo de construção de um poço de petróleo é da ordem de dezenas de milhões de reais. Existem diversas configurações de poços possíveis para o desenvolvimento de um campo e cada uma destas configurações possui características diferentes, como custo, produtividade, risco, número de zonas produtoras, etc.

Pena e Lohr (2017) avaliaram as melhores maneiras de se reduzir custos e riscos durante a execução de um projeto em águas ultra profundas. Eles apontaram que as maiores melhorias foram obtidas através de avanços nas complexidades dos poços e sistemas submarinos, nas trajetórias dos poços, da integração do projeto do poço com a completação e de melhoras na cadeia de fornecimento.

Diante disso, a escolha de qual configuração de poço será utilizada passa então a ser primordial para o sucesso econômico na exploração de um campo ou para a redução do brent de equilíbrio, preço mínimo do barril que torna a exploração do campo viável. A escolha da configuração é, no entanto, uma tarefa complexa e que envolve times multidisciplinares (geologia, reservatório, elevação e escoamento, sistemas submarinos, perfuração, completação, etc.).

O processo de escolha da melhor configuração envolve um trabalho inicial dos projetistas de poços que elaboram várias alternativas, das quais, diversas são eliminadas nas fases iniciais do projeto devido a não atenderem requisitos básicos do projeto. As alternativas restantes devem ser levadas para este time multidisciplinar, e é realizado um workshop de seleção de poços. Neste workshop é aplicado o conceito de competitive 
scoping, na qual as alternativas competem umas com as outras, sendo analisadas sob diversos critérios.

Como a escolha da configuração que será utilizada para o desenvolvimento do campo envolve múltiplos critérios, a aplicação de ferramentas de apoio multicritério à decisão (AMD) pode auxiliar o grupo de decisores a realizarem a ordenação dos melhores projetos baseados em critérios cujos pesos podem depender do objetivo e do cenário sendo avaliado.

Num cenário de redução de custos para garantir a viabilidade econômica de um campo ou na exploração de um campo maduro, o critério de custo pode ser definido como tendo maior importância, ao passo que num projeto objetivando “ramp up”, ou seja, aumento da produção do campo, a produtividade do poço pode ser definida como critério mais importante.

A motivação deste trabalho é aplicar uma técnica de apoio multicritério à decisão para a auxiliar na priorização das melhores configurações de poços para o desenvolvimento de um campo. Para tanto será aplicado uma técnica hibrida AHP-TOPSIS-2N num workshop de seleção de configurações de poços de uma empresa petrolífera brasileira. Serão avaliados dois cenários distintos, um cenário de redução de custo para o desenvolvimento do campo e um cenário de aumento do fator de recuperação do campo.

Este artigo é relevante pois apresenta um caso real de aplicação numa companhia petrolífera, considera diferentes cenários e objetivos para o desenvolvimento do campo. Além disso, este trabalho se insere num momento em que a redução do preço do petróleo dos últimos anos levou as operadoras a buscarem soluções otimizadas diante de incertezas e complexidades do mundo real.

O artigo está organizado da seguinte forma: a Seção 2 apresenta o problema de seleção de configurações de poços em detalhes, com os critérios mais relevantes a serem observados e a aplicação do método AHP-TOPSIS-2N com seus passos; A Seção 3 apresenta o estudo de caso; A Seção 4 apresenta os resultados. A Seção final fornece as discussões e conclusões do estudo.

\section{FUNDAMENTAÇÃO TEORICA}

Pledge et. al. (2017) afirma que desenvolvimento em águas ultra profundas é viável em ambientes de baixo preço do barril de petróleo. Os autores apresentam no artigo uma abordagem para projetar e executar um desenvolvimento de sistema de baixo custo para águas ultra profundas. Como resultado obtiveram uma redução entre 15 a $25 \%$ somente com reduções de escopo.

Pode-se pensar em duas abordagens para se chegar na escolha de uma configuração ou escopo para os poços de petróleo que serão construídos para a exploração de um campo, uma metodologia convencional e a metodologia do competitive scoping.

Na metodologia convencional, em linhas gerais, estabelece-se uma configuração de poço com todos os equipamentos desejáveis tanto para a performance do poço quanto para a segurança e integridade do mesmo, para então otimizar esta configuração preliminar. A nova metodologia consiste na abordagem competitive scoping, que parte de uma configuração 
mínima e a partir desta desenvolve novos escopos que atendam determinados objetivos (PLEDGE et al., 2017). A Figura ilustra como as duas abordagens caminham em direção oposta..Na Seção 2.1 será detalhado este novo processo de seleção de configuração de poços.

Figura 1 - Diferença entre a abordagem clássica e a abordagem do competitive scoping

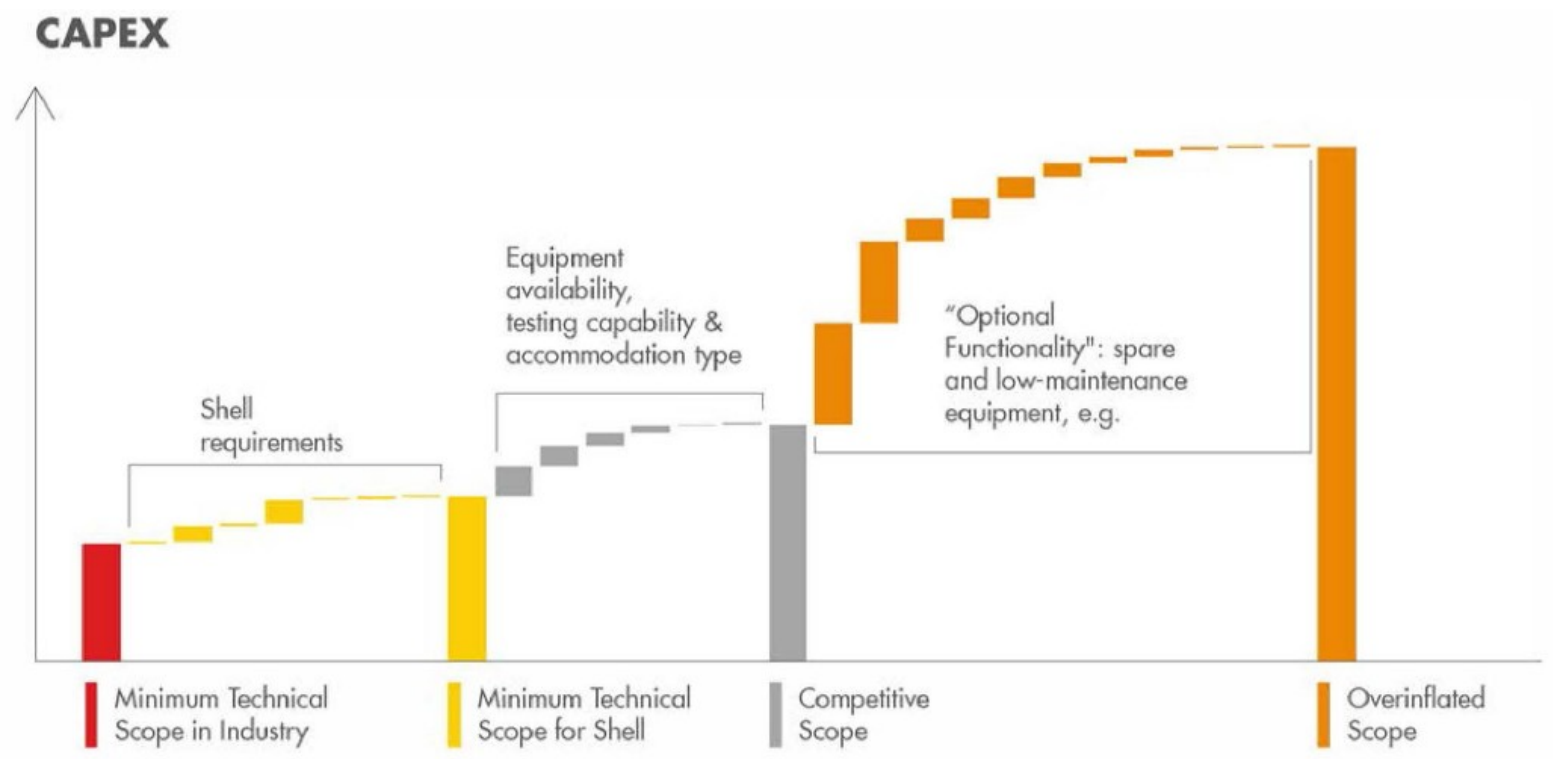

Fonte: Pledge et. al. (2017)

Como a nova metodologia consiste na escolha otimizada entre diversas alternativas de configurações disponíveis e existem diversos critérios, conflitantes entre si, para determinar a escolha ótima, o uso de métodos de apoio multicritério a decisão podem contribuir neste processo. A seção 2.2 descreve no que se constituem tais métodos e a Seção 2.2.1 descreve o método empregado no estudo: o AHP-TOPSIS-2N.

\subsection{SELEÇÃO DE CONFIGURAÇÃO DE POÇOS}

Uma empresa brasileira está migrando para uma nova metodologia de seleção de configurações, em substituição a metodologia convencional, objetivando a busca de uma configuração mais otimizada de poços. O processo de seleção das configurações de poços, que a companhia em estudo está implementando, utiliza uma sistemática de competitive scope que possui as seguintes etapas:

- Objetivo do projeto

- Requisitos mínimos de projeto

- Seleção das configurações de poços

- Estimação da duração e custo

- Avaliação do risco operacional, risco tecnológico e produtividade para cada configuração

- Redução para um número menor de alternativas que atendam aos objetivos, requisitos e limites mínimos e máximos para os critérios

- Workshop de seleção de alternativas de configuração de poços 
A primeira etapa da metodologia consiste em determinar qual será o objetivo a ser alcançado. Em via de regra, esse objetivo será um resultado econômico, no entanto pode vir a ser um determinado nível de produção, ou o desenvolvimento de uma nova tecnologia. A elaboração de requisitos mínimos de projeto envolve um entendimento e caracterização da área do campo produtor. Características como pressão e temperatura do reservatório, presença de contaminantes como $\mathrm{CO}_{2}$ e $\mathrm{H}_{2} \mathrm{~S}$, espessura do reservatório, permeabilidade etc., são importantes para a definição destes requisitos.

Após a determinação dos requisitos, é feita uma análise, onde se determina a configuração de poço mais simples e enxuta possível, que atenda aos critérios de segurança e requisitos mínimos levantados anteriormente. Uma vez determinada a configuração de poço mais exígua, propõe-se a configuração seguinte incorporando a menor modificação possível que gere diminuição do risco de falha do poço e/ou ganho de produtividade. Seguindo esta rotina reiteradamente, são geradas diferentes configurações de poços, formando um espectro de arquiteturas das mais simples às mais complexas que minimizam o risco de falha do poço e maximizam a sua produção.

Uma configuração de poço envolve todos os detalhes de sua construção, número de fases, tipos de fluidos utilizados, número de revestimentos, tipos de coluna de completação, entre outros. Como não é o escopo deste estudo, explorar os detalhes de cada configuração, será apresentado três características fundamentais para a definição da configuração do poço.

A primeira característica destacada é o diâmetro da fase final do poço de petróleo, pois influencia a área de exposição ao reservatório e, portanto, a produtividade final do mesmo. Poços com maiores diâmetros tendem a ter uma produção maior (RAHIM et. al., 2015), porém, são mais complexos e, geralmente, apresentam um custo maior de construção. Outra característica importante é se na última fase do poço, aquela exposta ao reservatório, o poço é revestido e canhoneado ou é deixado aberto. Na Erro: Origem da referência não encontrada pode ser visto um poço aberto, poço da esquerda, e um poço revestido e canhoneado, poço da direita. No poço aberto, a área de fluxo é muito maior, porém, o mesmo pode apresentar alguns problemas, como produção de sólidos, colapso, entre outros. O poço revestido tem um revestimento metálico que protege as paredes do poço, tornando-o mais caro e reduzindo a área de fluxo, limitada aos furos produzidos pelo canhoneio. 
Figura 2 - Poço aberto e poço revestido
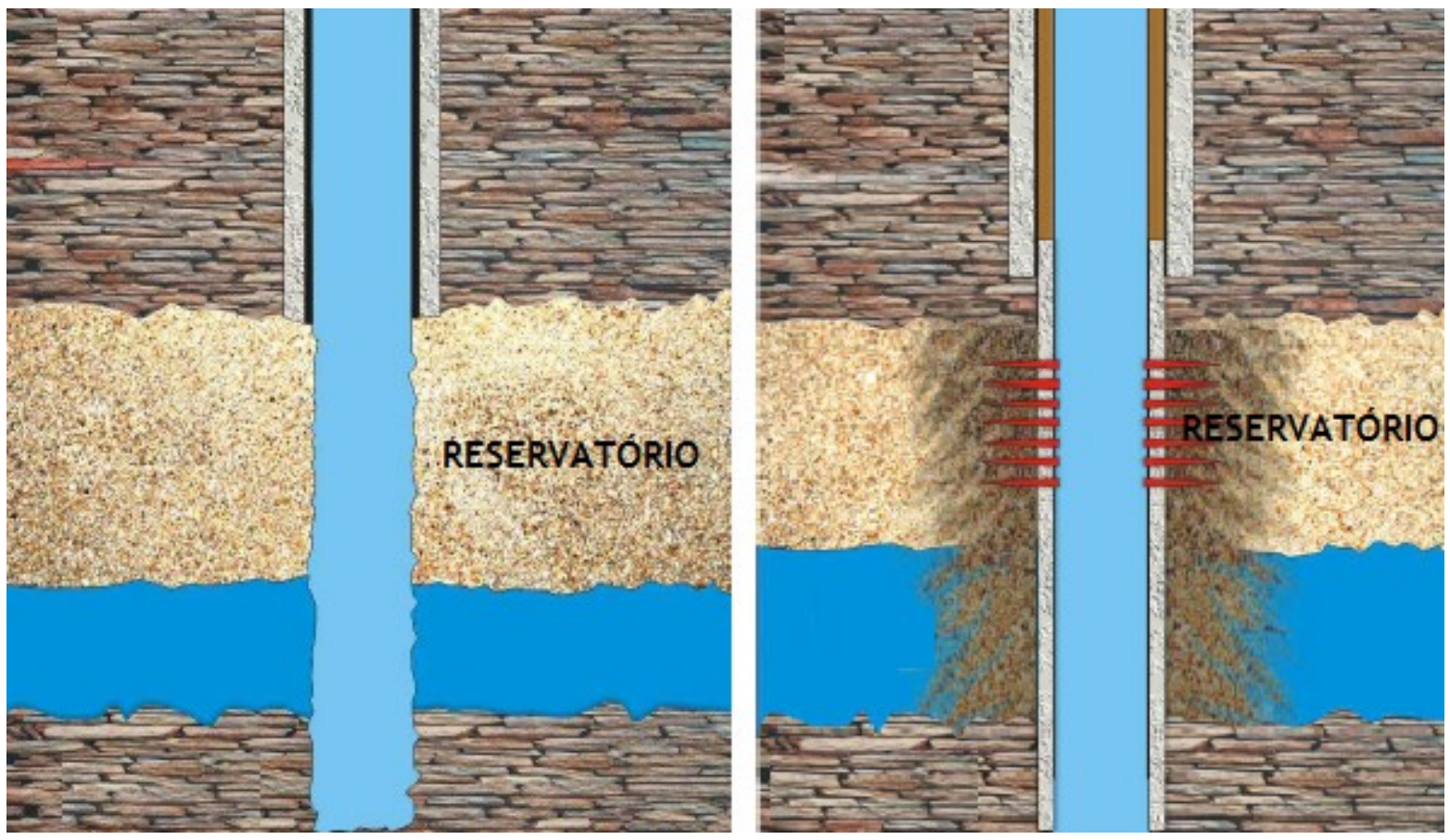

Em relação a completação do poço, pode-se destacar dois tipos: os poços de completação simples e os poços de completação inteligente, Erro: Origem da referência não encontrada. Os poços de completação simples produzem pela extremidade da coluna e conseguem produzir de apenas uma única zona, não possuindo seletividade. Já os poços de completação inteligente possuem válvulas acionadas por linhas hidráulicas que permitem a produção de diversas zonas, usualmente duas ou três, de forma seletiva. A completação inteligente permite um maior gerenciamento da produção, em especial nos campos do pré-sal que possuem reservatórios com zonas de propriedades distintas. Além disso, a completação inteligente permite um maior monitoramento.

A completação inteligente envolve um maior custo, tanto pela aquisição destas válvulas, quanto pela própria operação de instalação das mesmas ser mais demorada. Além disso, válvulas de determinados diâmetros ou outros equipamentos podem requerer algum grau de desenvolvimento tecnológico, resultando num risco tecnológico alto. 
Figura 2- Poço de completação simples e poço de completação inteligente
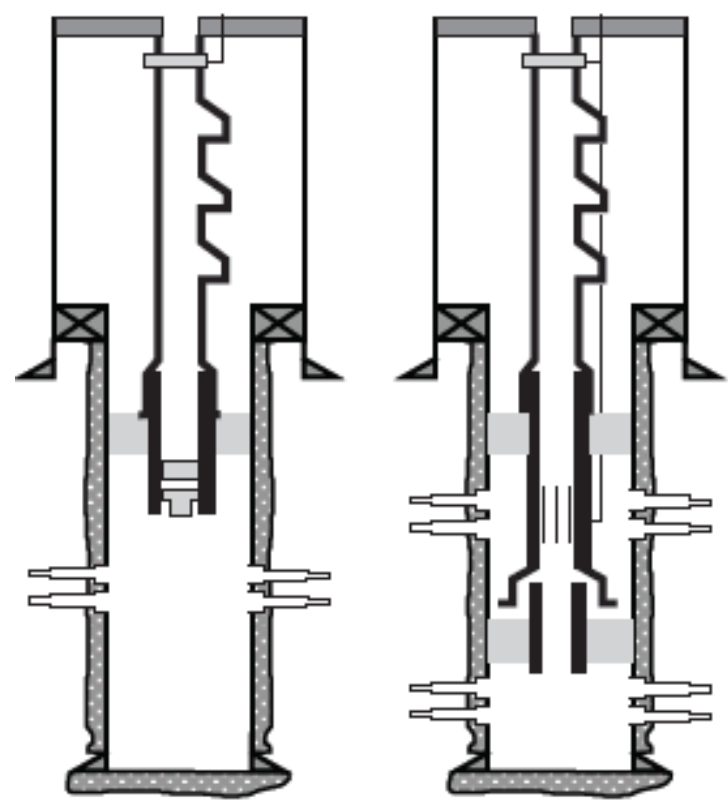

A construção de um poço de petróleo envolve duas etapas, a perfuração, na qual a rocha é removida por uma broca e o poço é aberto, e a completação, na qual o poço é devidamente equipado com uma coluna de produção, válvulas e outros equipamentos. A estimativa de duração de cada uma destas fases é feita por meio da sequência operacional e da análise histórica de duração de cada operação em outros poços. A análise da duração é fundamental, pois o custo de construção do poço é praticamente proporcional a sua duração, dado que o principal custo envolvido é o valor de diária da sonda marítima utilizada para construir o poço. Existe um valor de custo que inviabiliza a execução financeira do projeto, e as configurações que ultrapassam este valor são eliminadas da análise.

O risco operacional fornece uma medida do risco de ocorrência de alguma falha (NURAFZA et. al., 2018), que pode evoluir para um acidente durante a construção e operação do poço, sendo o principal deles o blowout, ou o vazamento descontrolado de hidrocarbonetos para o meio ambiente. A metodologia de cálculo do fator risco operacional é complexa e envolve várias dimensões como: logística da locação, condições ambientais, necessidade de workovers, risco de perda de circulação etc. O risco tecnológico é uma medida da probabilidade de insucesso em ter disponível todas as tecnologias necessárias à construção do poço na data de sua execução. O cálculo depende da quantidade de equipamentos necessários para a configuração do poço e o grau de maturidade tecnológica, TRL (MANKINS, 1995) (Technology Readiness Level), de cada um.

\subsection{MODELO DE APOIO MULTICRITÉRIO A DECISÃo PARA PRIORIZAR AS CONFIGURAÇÕES}

O processo de escolha da melhor alternativa de configuração de poço envolve diversos critérios, conflitantes entre si, e um grupo multidisciplinar. Nestas situações, é intrínseco do ser humano o desejo de alcançar seus objetivos em detrimento dos objetivos grupais (MORAIS E FONTANA, 2019). Neste contexto, a proposição de métodos que auxiliem no processo de tomada de decisão é fundamental (GOMES et. al., 2013). Métodos de Apoio à 
Decisão (AMD) são aplicados quando se tem a necessidade de selecionar, ordenar, classificar ou descrever alternativas presentes em processo decisório complexo com múltiplos critérios e objetivos em conflito (SOUZA et. al., 2016). A escolha do método AMD a ser utilizada depende das características do problema e, principalmente, do objetivo dos decisores (FONTANA e HOLANDA, 2019).

\subsubsection{Método AHP-TOPSIS-2N}

O Método AHP-TOPSIS-2N é um método híbrido constituído por duas técnicas de tomada de decisão multicritério que são usualmente adotadas em cenários complexos, caracterizados pela presença de objetivos múltiplos e conflitantes. Para entender o método é necessário um entendimento prévio das duas técnicas que o compõe.

O Método AHP (abreviação do inglês Analytic Hierarchy Process) foi desenvolvido por Thomas Saaty na década de 1970 (SAATYy, 1980). Trata-se de uma técnica quantitativa para o problema de MCDM (Multi Criteria Decision Making), sendo uma das mais utilizadas devido a sua facilidade e por ser intuitiva. O método é utilizado para ponderar os pesos dos critérios, comparando-os dois a dois segundo uma escala, como ilustrado na Tabela 1. Para garantir a consistência da decisão, o método AHP dispõe do recurso que calcula as denominadas Razões de Consistência (RC) entre o Índice de Consistência (IC) dos julgamentos e o Índice de Consistência Randômico (IR). Para garantir um nível aceitável, a RC deve estar abaixo de 10\%, considerada tolerância máxima de inconsistência. O peso para cada critério é calculado através do autovetor normalizado da matriz de ponderação.

Tabela 1 - Escala Fundamental de Saaty. Fonte: Saaty (1980)

\begin{tabular}{|c|c|c|}
\hline VALOR & DEFINIÇÃO & EXPLICAÇÃO \\
\hline 1 & Igual importância & $\begin{array}{l}\text { os dois critérios contribuem de forma idêntica para } \\
\text { o objetivo }\end{array}$ \\
\hline 3 & $\begin{array}{l}\text { Pouco mais impor- } \\
\text { tante }\end{array}$ & $\begin{array}{l}\text { a análise e a experiência mostram que um critério } \\
\text { é um pouco mais importante que o outro }\end{array}$ \\
\hline 5 & $\begin{array}{l}\text { Muito mais impor- } \\
\text { tante }\end{array}$ & $\begin{array}{l}\text { a análise e a experiência mostram que o critério é } \\
\text { claramente mais importante que o outro }\end{array}$ \\
\hline 7 & $\begin{array}{l}\text { Bastante mais im- } \\
\text { portante }\end{array}$ & $\begin{array}{l}\text { a análise e a experiência mostram que um dos } \\
\text { critérios é predominante para o objetivo }\end{array}$ \\
\hline 9 & $\begin{array}{l}\text { Extremamente mais } \\
\text { importante }\end{array}$ & $\begin{array}{l}\text { sem qualquer dúvida um dos critérios é absoluta- } \\
\text { mente predominante para o objetivo }\end{array}$ \\
\hline $\begin{array}{c}\mathbf{2 , 4 , 6 , 8} \\
\text { valores recíprocos dos anteriores }\end{array}$ & $\begin{array}{l}\text { Valores intermediá- } \\
\text { rios }\end{array}$ & também podem ser utilizados \\
\hline
\end{tabular}

O Método TOPSIS (abreviação do inglês Technique for Order Preferences by Similarity to Ideal Solution) é uma outra técnica para MCDM e foi apresentada por Hwang e Yoon em 1981 (HWANG e YOON, 1981). Este método se destaca por ser intuitivo e pela simplicidade dos procedimentos matemáticos, facilidade de implementação e aplicação, e permite avaliar uma quantidade não limitada de alternativas (SOUZA et. al.,2016). Para o TOPSIS, a melhor alternativa é aquela que está mais próxima da solução ideal positiva e a mais distante da solução ideal negativa. 
A solução ideal positiva é aquela que maximiza os critérios “positivos” e minimiza os critérios "negativos”. A solução ideal negativa é a oposta, ou seja, maximiza os critérios “negativos” e minimiza os critérios "negativos”.

Alguns autores vêm utilizando métodos que integram ambas as técnicas. Prakash e Barua (PRAKASH e BARUA, 2015) aplicaram uma integração AHP-TOPSIS para avaliar e priorizar estratégias de logística reversa em um ambiente com incertezas. Utilizaram também técnica fuzzy para aumentar a robustez. Souza et. al. (SOUZA et. al.,2016) utilizou um método hibrido composto pelas técnicas AHP, TOPSIS e também a agregação dos resultados dos decisores com AIP (Agregação Individual de Prioridades). Caiado et. al. (2018) aplicaram o método AHP-TOPSIS para classificar as alternativas de veículo leves quanto ao ecoeficiência. Souza et. al. (SOUZA et. al, 2018) implementaram um método híbrido de AHP e TOPSIS com duas normalizações, chamando de AHP-TOPSIS-2N, e o aplicaram na priorização de portfólios de projetos de investimento na área de TI.

Os resultados obtidos em (SOUZA et. al., 2018) mostraram que o método AHPTOPSIS-2N tem grande potencial para lidar com problemas de priorização de projetos de investimento, como é o caso da escolha da melhor alternativa de projeto de poço para o desenvolvimento de um campo de petróleo offshore. Os passos para a aplicação do método em questão são descritos a seguir:

1. Definição da Matriz de Decisão: Expressa a pontuação de cada alternativa em relação a cada critério, mostrando o quanto uma alternativa domina ou é dominada pelas demais;

2. Definição da Matriz de Ponderação: utilizando a escala fundamental de Saaty, através de grupo multidisciplinar cada critério é avaliado contra cada critério;

3. Determinação do peso de cada critério: através da aplicação do método AHP. É importante avaliar a razão de consistência e caso a mesma seja superior a 0,1 fazer uma nova avaliação dos critérios e uma nova matriz de ponderação;

4. Normalização da Matriz de Decisão: no caso do método AHP-TOPSIS-2N são utilizadas duas diferentes normalizações (SOUZA et. al., 2018).

5. Construção da Matriz de Decisão Normalizada Ponderada: as matrizes ponderadas pelo $1^{\circ}$ e $2^{\circ}$ normalização são ponderadas utilizando os pesos obtidos no passo 3;

6. Determinação da Solução Ideal Positiva (SIP) e da Solução Ideal Negativa (SIN);

7. Cálculo das medidas de distância: são obtidas medidas de distância euclidiana de cada uma das alternativas em relação a SIP e a SIN;

8. Cálculo da proximidade relativa à alternativa ideal: é obtida pela razão $\mathrm{R}$ igual a distância para o ponto SIN pela soma da distância ao ponto SIP e a distância para o ponto SIN;

9. Ordenação das preferências. 


\section{ESTUDO DE CASO}

Para o estudo de caso foram escolhidas 4 configurações de 20 possíveis para o desenvolvimento de um campo petrolífero do pré-sal. Estas 4 configurações foram préselecionadas pelo grupo multidisciplinar após eliminar aquelas que não atendiam aos objetivos ou requisitos mínimos do projeto.

A seguir seguem algumas características genéricas das configurações selecionadas. O detalhamento de cada configuração não pode ser exposto no artigo pois trata-se de elemento diferencial competitivo da empresa em questão.

- Configuração A: Trata-se de uma configuração de poço aberto, ou seja, não revestido e com completação inteligente para a produção de até 3 zonas. Trata-se de um poço de pequeno diâmetro.

- Configuração B: Trata-se de uma configuração de poço aberto, ou seja, não revestido, com completação simples, ou seja, produz de apenas 1 zona. Apesar de ser um poço de pequeno diâmetro, tem uma coluna de produção de grande diâmetro.

- Configuração C: Trata-se de um poço revestido e canhoneado, com completação inteligente para a produção de duas zonas. É um poço de grande diâmetro.

- Configuração D: Trata-se um poço aberto, ou seja, não revestido, com completação inteligente para três zonas. Trata-se de um poço de grande diâmetro e com coluna de produção de grande diâmetro também.

Para avaliar a melhor das quatro alternativas foram definidos 5 critérios:

- C1- Custo (Custo): medido pelo custo estimado de construção do poço em milhões de dólares

- C2- Produtividade (Prod.): medido pela vazão esperada do poço em milhares de barris por dia

- C3- Risco Operacional (RiscOper.): número real de 0 a 10 determinado através de uma matriz de risco

- C4- Risco Tecnológico (RiscTec.): número real entre 0 e 5 determinado através da análise de maturidade das tecnologias envolvidas

- C5- Número de Zonas Produtoras (NumZonas): número natural que indica a quantidade de zonas produtoras que podem ser gerenciadas pelo poço

A matriz de avaliação das alternativas de configurações de poços em razão dos critérios definidos pode ser vista na Tabela 2 .

Tabela 2 - Matriz de Decisão

\begin{tabular}{l|ccccc} 
& $\begin{array}{c}\text { Custo } \\
\text { (MM US\$) }\end{array}$ & $\begin{array}{c}\text { Prod. } \\
\text { (Kbpd) }\end{array}$ & $\begin{array}{c}\text { RiscO- } \\
\text { per. }\end{array}$ & RiscTec. & NumZonas \\
\hline CONFIGURAÇÃO A & 114 & 66 & 2,3 & 2,5 & 3 \\
CONFIGURAÇÃO B & 102,8 & 30 & 3,1 & 2,1 & 1 \\
CONFIGURAÇÃO C & 118,4 & 70 & 4,05 & 3,1 & 2 \\
CONFIGURAÇÃO D & 126,6 & 99 & 7,3 & 3,6 & 3
\end{tabular}


Para avaliar a importância relativa dos critérios foram considerados dois cenários para o desenvolvimento do campo:

- Cenário 1: O objetivo seria desenvolver o campo com o menor custo possível de forma que o brent de equilíbrio tivesse valor compatível com as previsões pessimistas para o preço do barril no futuro. Além disso, prioriza-se neste caso a redução dos riscos operacionais, pois a ocorrência de um acidente, como o vazamento de um poço pode gerar facilmente um VPL negativo para o projeto.

- Cenário 2: O objetivo seria acelerar a curva de produção e aumentar o fator de recuperação do campo, ou seja, extrair o máximo de petróleo e acelerar a produção. A vazão dos poços é importante para atingir estes objetivos, porém, o número de zonas produtoras para cada poço é importante também para o gerenciamento do reservatório.

Na prática, para o desenvolvimento de um campo de petróleo, pode-se utilizar mais de uma configuração para atingir um cenário combinado (1 e 2). Um exemplo seria a utilização de uma configuração de poço para os primeiros a serem construídos, objetivandose um rápido crescimento da produção e com isso topar a plataforma, e posteriormente, e outra configuração para os poços seguintes, com o objetivo de manter a produção com um baixo custo. Com base nisso, para cada um dos cenários foi construída uma matriz de ponderação dos critérios, conforme Tabela 3 e Tabela 4.

Tabela 3- Matriz de Ponderação para o Cenário 1

\begin{tabular}{|c|c|c|c|c|c|}
\hline & Custo & Prod. & $\begin{array}{c}\text { Risco- } \\
\text { per. }\end{array}$ & RiscTec. & NumZonas \\
\hline CUSTO & 1 & 7 & 3 & 9 & 9 \\
\hline PRODUTIVIDADE & $1 / 7$ & 1 & 1 & 5 & 7 \\
\hline RISCO OPERACIONAL & $1 / 3$ & 1 & 1 & 5 & 7 \\
\hline RISCO TECNOLOGICO & $1 / 9$ & $1 / 5$ & $1 / 5$ & 1 & 1 \\
\hline NÚMERO DE ZONAS & $1 / 9$ & $1 / 7$ & $1 / 7$ & 1 & 1 \\
\hline
\end{tabular}

Tabela 4 - Matriz de Ponderação para o Cenário 2

\begin{tabular}{c|ccccc}
\multicolumn{1}{c}{} & Custo & Prod. & $\begin{array}{c}\text { Risco- } \\
\text { per. }\end{array}$ & RiscTec. & NumZonas \\
\hline CUSTO & 1 & $1 / 5$ & 5 & 3 & $1 / 5$ \\
PRODUTIVIDADE & 5 & 1 & 9 & 9 & 3 \\
RISCO OPERACIONAL & $1 / 5$ & $1 / 9$ & 1 & $1 / 5$ & $1 / 7$ \\
RISCO TECNOLOGICO & $1 / 3$ & $1 / 9$ & 5 & 1 & $1 / 7$ \\
NÚMERO DE ZONAS & 5 & $1 / 3$ & 7 & 7 & 1
\end{tabular}

\section{RESULTADOS}


O resultado da metodologia AHP para gerar o peso de cada critério em cada um dos cenários está representado na Tabela 5.

Tabela 5 - Pesos dos Critérios para cada um dos Cenários

\begin{tabular}{c|cc|cc|} 
& \multicolumn{2}{|c|}{ CENÁRIO 1 } & \multicolumn{2}{c|}{ CENÁRIO 2 } \\
& RANKING & PESO & RANKING & PESO \\
\hline CUSTO & 1 & 0,5482 & 3 & 0,1105 \\
PRODUTIVIDADE & 3 & 0,1708 & 1 & 0,5068 \\
RISCO OPERACIONAL & 2 & 0,2024 & 5 & 0,0281 \\
RISCO TECNOLOGICO & 4 & 0,0419 & 4 & 0,0592 \\
NÚMERO DE ZONAS & 5 & 0,0366 & 2 & 0,2953
\end{tabular}

O resumo das consistências dos cenários é apresentado na Tabela 6

Tabela 6 - Consistência da Matriz de Ponderação dos Critérios

\begin{tabular}{c|c|c|} 
& CENÁRIO 1 & CENÁRIO 2 \\
\hline LAMBDA MAX & 5,4031 & 5,4433 \\
INDICE DE CONSISTÊNCIA & 0,1008 & 0,1108 \\
RAZÃO DE CONSISTÊNCIA & 0,09 & 0,099
\end{tabular}

Como as razões de consistência para ambos os cenários foram menores do que 0,01, pode-se concluir que as matrizes de ponderação dos critérios são consistentes.

O resultado da aplicação das duas normalizações para a matriz de decisão pode ser visto nas Tabela 7 e Erro: Origem da referência não encontrada.

Tabela 7 - Matriz de Peso Ponderada para a $1^{\circ}$ Normalização nos Cenários 1 e 2

\begin{tabular}{|c|c|c|c|c|c|}
\hline \multicolumn{6}{|c|}{1 Normalização } \\
\hline & CUSTO & PROD. & RISCOPER. & RISCTEC. & NUMZONAS \\
\hline CONFIGURAÇÃO A & 0,4924 & 0,4672 & 0,2501 & 0,4337 & 0,6255 \\
\hline CONFIGURAÇÃO B & 0,4440 & 0,2124 & 0,3371 & 0,3643 & 0,2085 \\
\hline CONFIGURAÇÃO C & 0,5114 & 0,4955 & 0,4403 & 0,5378 & 0,4170 \\
\hline CONFIGURAÇÃO D & 0,5468 & 0,7937 & 0,7937 & 0,6245 & 0,6255 \\
\hline \multicolumn{6}{|c|}{ Ponderada para Cenário 1} \\
\hline & CUSTO & PROD. & RISCOPER. & RISCTEC. & NUMZONAS \\
\hline CONFIGURAÇÃO A & 0,2699 & $\begin{array}{c}0,079 \\
8\end{array}$ & 0,0506 & 0,0182 & 0,0229 \\
\hline CONFIGURAÇÃO B & 0,2434 & $\begin{array}{c}0,036 \\
3\end{array}$ & 0,0682 & 0,0153 & 0,0076 \\
\hline CONFIGURAÇÃO C & 0,2803 & $\begin{array}{c}0,084 \\
6\end{array}$ & 0,0891 & 0,0225 & 0,0153 \\
\hline CONFIGURAÇÃO D & 0,2998 & $\begin{array}{c}0,119 \\
7\end{array}$ & 0,1606 & 0,0262 & 0,0229 \\
\hline
\end{tabular}


Ponderada para Cenário 2

\begin{tabular}{l|ccccc}
\hline & CUSTO & PROD. & RISCOPER. & RISCTEC. & NUMZONAS \\
\hline CONFIGURAÇÃO A & 0,0544 & $\begin{array}{c}0,236 \\
8\end{array}$ & 0,0070 & 0,0257 & 0,1847 \\
CONFIGURAÇÃO B & 0,0491 & $\begin{array}{c}0,107 \\
6\end{array}$ & 0,0095 & 0,0216 & 0,0616 \\
CONFIGURAÇÃO C & 0,0565 & $\begin{array}{c}0,251 \\
1\end{array}$ & 0,0124 & 0,0318 & 0,1231 \\
CONFIGURAÇÃO D & 0,0604 & 0,355 & 0,0223 & 0,0370 & 0,1847
\end{tabular}

Tabela 8- Matriz de Peso Ponderada para a $2^{\circ}$ Normalização nos Cenários 1 e 2

2 №rmalização

\begin{tabular}{l|ccccc} 
& CUSTO & PROD. & RISCOPER. & RISCTEC. & NUMZONAS \\
\hline CONFIGURAÇÃO A & 0,4706 & 0,5217 & 0,0000 & 0,2667 & 1,0000 \\
CONFIGURAÇÃO B & 0,0000 & 0,0000 & 0,1600 & 0,0000 & 0,0000 \\
CONFIGURAÇÃO C & 0,6555 & 0,5797 & 0,3500 & 0,6667 & 0,5000 \\
CONFIGURAÇÃO D & 1,0000 & 1,0000 & 1,0000 & 1,0000 & 1,0000
\end{tabular}

Ponderada para Cenário 1

\begin{tabular}{l|ccccc}
\hline & CUSTO & PROD. & RISCOPER. & RISCTEC. & NUMZONAS \\
\hline CONFIGURAÇÃO A & 0,2580 & 0,089 & 0,0000 & 0,0112 & 0,0366 \\
CONFIGURAÇÃO B & 0,0000 & $\begin{array}{c}0,000 \\
0\end{array}$ & 0,0324 & 0,0000 & 0,0000 \\
CONFIGURAÇÃO C & 0,3593 & 0,099 & 0,0708 & 0,0279 & 0,0183 \\
CONFIGURAÇÃO D & 0,5482 & $\begin{array}{c}0,170 \\
8\end{array}$ & 0,2024 & 0,0419 & 0,0366
\end{tabular}

Ponderada para Cenário 2

\begin{tabular}{l|ccccc}
\hline & CUSTO & PROD. & RISCOPER. & RISCTEC. & NUMZONAS \\
\hline CONFIGURAÇÃO A & 0,0520 & $\begin{array}{c}0,264 \\
4\end{array}$ & 0,0000 & 0,0158 & 0,2953 \\
CONFIGURAÇÃO B & 0,0000 & $\begin{array}{c}0,000 \\
0\end{array}$ & 0,0045 & 0,0000 & 0,0000 \\
CONFIGURAÇÃO C & 0,0724 & $\begin{array}{c}0,293 \\
8\end{array}$ & 0,0098 & 0,0395 & 0,1476 \\
CONFIGURAÇÃO D & 0,1105 & $\begin{array}{c}0,506 \\
8\end{array}$ & 0,0281 & 0,0592 & 0,2953
\end{tabular}

Por fim, o resultado de priorização pode ser visto na Tabela 9. 
Tabela 9 - Priorização das alternativas de configuração de poços para os dois cenários e os dois processos de normalização

\begin{tabular}{|c|c|c|c|c|c|c|c|c|}
\hline \multirow[b]{3}{*}{ 10 COLOCADA } & \multicolumn{4}{|c|}{ CENÁRIO 1} & \multicolumn{4}{|c|}{ CENÁRIO 2} \\
\hline & \multicolumn{2}{|c|}{ 1N NORM } & \multicolumn{2}{|c|}{ 2NORM } & \multicolumn{2}{|c|}{ 1NORM } & \multicolumn{2}{|c|}{ 2NORM } \\
\hline & A & $\begin{array}{c}0,719 \\
6\end{array}$ & B & 0,7641 & $\mathrm{D}$ & $\begin{array}{c}0,918 \\
6\end{array}$ & $\mathrm{D}$ & 0,8203 \\
\hline 2 COLOCADA & C & $\begin{array}{c}0,578 \\
8\end{array}$ & A & 0,5760 & A & $\begin{array}{c}0,602 \\
3\end{array}$ & A & 0,6193 \\
\hline 30 COLOCADA & B & $\begin{array}{c}0,556 \\
8\end{array}$ & C & 0,4018 & C & $\begin{array}{c}0,562 \\
7\end{array}$ & C & 0,5496 \\
\hline 4 COLOCADA & D & $\begin{array}{c}0,405 \\
9\end{array}$ & D & 0,2297 & B & $\begin{array}{c}0,076 \\
8\end{array}$ & B & 0,1787 \\
\hline
\end{tabular}

\section{DISCUSSÕES E CONCLUSÃO}

Nota-se que a escolha da melhor configuração de poço para o desenvolvimento de um campo é altamente dependente dos objetivos e requisitos para o projeto de desenvolvimento, como pode ser visualizado nos resultados de ordenação obtidos para os diferentes cenários. A Configuração D que ficou em último lugar em ambas normalizações para o Cenário 1, foi a primeira colocada no Cenário 2. O estudo demonstrou a importância que deve ser dada a elaboração e caracterização do cenário previamente a escolha das configurações.

A definição dos cenários impacta na ponderação dos critérios dada pela importância relativa de um critério em relação ao outro. No Cenário 1, o critério de custo teve um peso de mais de $50 \%$, dado que o objetivo do projeto é justamente reduzir este componente. O segundo critério foi o risco operacional, o que faz sentido, considerando que um acidente que leve ao vazamento de um poço, pode inviabilizar completamente a economicidade do projeto. A produtividade também é um fator importante, pois caso o poço possua uma produtividade maior, podem-se construir menos poços, e com isso reduzir o custo total do projeto.

No Cenário 2, o critério de produtividade teve um peso de mais de 50\% pela mesma razão anterior, trata-se do objetivo do projeto que é aumentar a produção. O segundo critério foi o número de zonas, mostrando que a capacidade de gerenciar várias zonas do reservatório é importante para se atingir a produtividade e fator de recuperação adequados. O risco tecnológico teve sua importância superior ao risco operacional, pois para atender os requisitos anteriores é necessário o desenvolvimento de válvulas inteligentes e de grande diâmetro. Caso estes desenvolvimentos não ocorram conforme o planejado, pode-se atrasar ou inviabilizar o projeto destes poços.

Sobre a ordenação das alternativas, no Cenário 1, onde se objetiva o desenvolvimento do campo com um menor custo, garantindo viabilidade econômica do projeto, a alternativa $\mathrm{D}$ foi a última colocada em ambas as normalizações, desta forma, ela poderia ser eliminada sem preocupação. A Configuração A ficou nas posições $1^{\circ}$ e $2^{\circ}$, somando 1,2956 de pontuação de desempenho. A Configuração B ficou em $1^{\circ}$ e $3^{\circ}$, somando 1,3209 de pontuação de desempenho. A Configuração B também é a que possui o menor custo entre as quatro possíveis. Sendo assim, ambas as configurações são escolhas plausíveis 
e cabe ao grupo de decisores avaliar qual delas melhor se enquadrará, podendo ser incluído algum novo critério para desempate ou feita uma reavaliação nos pesos dos critérios.

No caso do Cenário 2, como ambas as normalizações produziram os mesmos resultados de ordenação, o processo de escolha fica com uma robustez maior. O uso de dois processos de normalização se comporta como uma análise de sensibilidade, dando uma garantia maior na aplicação dos resultados. Neste caso a Configuração D se mostrou como a melhor alternativa. De fato, a alternativa D é um poço de grande diâmetro que favorece uma alta vazão e pode gerenciar a produção de 3 zonas ao mesmo tempo, tornando-o ideal para o cenário que se almeja alta produtividade.

Conclui-se que a nova abordagem de escolha de configuração de poços em relação ao processo convencional, pois favorece o estabelecimento de mais opções para os poços e avalia segundo diferentes objetivos. Da mesma forma, conclui-se que a utilização de um método híbrido de AMD auxilia a companhia a estabelecer a alternativa otimizada segundo os critérios definidos pelo grupo multidisciplinar de tomadores de decisão.

Como sugestão de trabalho futuro sugere-se que os cenários analisados sejam gerados utilizando uma metodologia de prospecção de cenários futuros, avaliando como cada uma das variáveis impacta em outras e com isso, determinar se as variáveis utilizadas como critérios são de fato as mais relevantes, se há necessidade de incluir alguma outra variável no estudo, ou até mesmo eliminar alguma. Também se sugere a avaliação do desenvolvimento do campo utilizando uma combinação de configurações que poderia otimizar ainda mais a escolha, obtendo-se assim um resultado superior ao da melhor configuração única.

\section{REFERÊNCIAS BIBLIOGRÁFICAS}

[1] Caiado, R.; Gomes, S.; Rangel, L.; Quelhas, O. (2016). Método Híbrido de Apoio à decisão AHP-TOPSIS para Avaliação de Ecoeficência de Transportes. XXX Congresso ANPET.

[2] Fontana, M.; Holanda, C. (2019). A multicriteria decision analysis on strategic operations in post-sale reverse logistics. Produção em Foco, 9(1), 73-97. https://doi.org/10.14521/P2237-5163.2019.0018.0004

[3] Gomez, J. (2013). On deciding how to decide: designing participatory budget processes. European Journal of Operational Reaserch, v.229, p.743-750. DOI: 10.1016/j.ejor.2013.03.035

[4] Hwang, C. L., Yoon, K.(1981). Multiple attributes decision Making Methods and Applications. Berlim: Springer.

[5] Mankins, J. (1995).Technology Readiness Levels, A White Paper. NASA, Washington, DC.

[6] Morais, D., Fontana, M., Silva, A. Lyra, M. (2019). Apoio à Decisão em Orçamento Participativo Estadual. Produção em Foco, 9 (1), 183-208. https://doi.org/10.14521/P2237-5163.2019.0018.0009

[7] Nurafza, P., Budhram, K., Julier, R.(2018). Development Well Risking of Production Forecasts. SPE Trinidad and Tobago Section Energy Resources Conference, Spain. 
[8] Pena, M., Lohr, C. Stones (2017). Development: Best in Class Risk Reduction and Cost Improvement During Project Execution. Offshore Technology Conference (OTC), Houston, Texas, USA. OTC-27850-MS.

[9] Pledge, D., Pereiras, A., Bryson, B., Orlean, R. (2017).Driving for Competing and Affordable Projects in the Gulf of Mexico. Offshore Technology Conference (OTC), Houston, Texas, USA. OTC-27947-MS.

[10] Prakash, C., Barua, M. K.(2015). Integration of AHP-TOPSIS method for prioritizing the solutions of reverse logistics adoption to overcome its barriers under fuzzy environment. Journal of Manufacturing Systems, v. 37 (3), pp. 599-615, ISSN 02786125, https://doi.org/10.1016/j.jmsy.2015.03.001

[11] Rahim, et. al. (2015) Analytical Solutions to Compute Optimal Well Configurations for Improved Gas Productivity. SPE Middle East Oil \& Gas Show and Conference, Bahrain. SPE-172529-MS Primeira referência. Primeira referência. Primeira referência. Primeira referência. Primeira referência.

[12] Saaty, T. L. (1980) Analytic Hierarchy Process. New York: McGraw Hill.

[13] Souza, L. P., Barros, A. P., Gomes, C. F. S. (2016) Comitê de Governança de TI: um modelo Decisório para Ordenamento e Priorização de Portfólio de Projetos de Investimento por Método Híbrido de Técnicas de Auxílio Multicritério, XIII SEGeT, Simpósio de Excelência em Gestão e Tecnologia.

[14] Souza, L.; Gomes, C. F. S.; Barros, A. (2018). Implementation of new Hybrid AHPTOPSIS-2N method in sorting and prioritizing of an it capex project portfolio. International Journal of Information Technology \& Decision Making. 10.1142/S0219622018500207. 